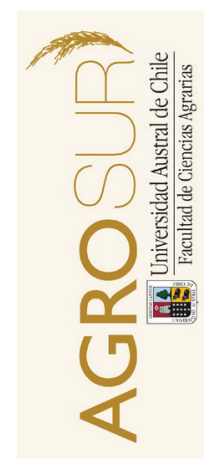

\title{
Estimación de la consanguinidad en un rebaño lechero doble propósito y su relación con rendimientos productivos y reproductivos: un estudio de caso en el Sur de Chile.
}

\author{
Estimation of inbreeding in a dual-purpose dairy herd and its relationship with \\ milk production and reproductive performance: A case study in Southern Chile.
}

\author{
Mujica, F. ${ }^{a *}$, Latrille, L. ${ }^{a}$, Vergara, $\mathrm{C}^{a}$ \\ ${ }^{a}$ Instituto de Producción Animal, Facultad de Ciencias Agrarias, Universidad Austral de Chile.
}

\begin{tabular}{l} 
A R T I C L E I N F O \\
\hline Keywords: \\
Inbreeding \\
Pedigree \\
Dairy herd \\
\hline Original Research Article, \\
Animal Science \\
\hline *Corresponding author: \\
Fernando Mujica \\
E-mail address: \\
fernandomujica@uach.cl
\end{tabular}

A B S T R A C T

The objective of the study was to estimate the degree of inbreeding in a commercial dairy herd of Red Dual Purpose Breed and its effects on number of days between calvings, age at first calving and milk production, using recorded data from 157 cows. The inbreeding index was calculated by using the software Pedigree Viewer, developed by Brian Kinghorn from the US Center for Livestock Genetic Evaluation. A file was created for each predigree, then every file was uploaded onto Pedigree Viewer to calculate the inbreeding coefficient on an individual basis. Twenty-four out of the 157 cow had some degree of inbreeding. The effect of inbreeding on number of days between calvings, age at first calving and milk production was then estimated but no effects were found. Results suggest that inbred cows might have a genetic superiority for the analyzed characteristics when compared with non-inbred cows. It is concluded that, at least in this case, the depression produced by inbreeding was balanced by the genetic superiority of the inbred cows.

\section{RESUMEN}

El objetivo de este trabajo, fue estimar el nivel de consanguinidad de un rebaño lechero, y sus efectos sobre el lapso interparto, la edad al primer parto y la producción de leche. El estudio se llevó a cabo con datos de un rebaño lechero de la raza Overo Colorado con datos provenientes de 157 vacas. Para el cálculo del coeficiente de endogamia se utilizó el programa Pedigree Viewer, desarrollado por Brian Kinghorn del Center for Genetic Evaluation of Livestock de Estados Unidos. Se elaboró un archivo por cada pedigrí y luego se ingresaron uno a uno al programa Pedigree Viewer para calcular el coeficiente de endogamia en forma individual. Se obtuvo que 24 de las 157 vacas, tenía algún nivel de consanguinidad. Posteriormente se evaluó el efecto de la consanguinidad sobre el lapso interparto, la edad al primer parto y la producción de leche, encontrándose que no tenía ningún efecto. De los resultados se podría deducir que las vacas consanguíneas presentarían una superioridad genética para las características analizadas al ser comparadas con las vacas que no tenían consanguinidad. Por lo tanto la depresión esperada causada por la consanguinidad, por lo menos en este caso, se habría contrarrestado con el efecto de la superioridad genética de las vacas consanguíneas.

Palabras clave: Endogamia, pedigrí, rebaño lechero.

\section{INTRODUCCIÓN}

En el manejo de los animales es de gran importancia, una vez seleccionados aquellos que participarán como reproductores, decidir el sistema de apareamiento que se implementará, el cual puede ser un cruzamiento consanguíneo (endocría) o cruzamiento de no parientes (exocría) (Mujica, 1992). El cruzamiento de individuos emparentados da origen a una progenie consanguínea, que presenta problemas en sus características reproductivas y productivas, como lo demuestran estudios realizados por MacNeil et al. (1992); Wiggans et al. (1995); Smith et al. (1998); Cassell (1999); Thompson et al. (2000a); Northcutt et al. (2001). Al mismo tiempo existe evidencia de que los niveles de consanguinidad están aumentando en los rebaños lecheros, lo que se atribuye a un mayor uso de la inseminación artificial, que utiliza muy pocos toros, ya que se eligen con mayor intensidad aquellos con un mayor mérito de acuerdo al objetivo productivo. Esto último acentúa posiblemente los efectos perjudiciales de este tipo de cruzamiento (Miglior y Burnside, 1995; Kuchida et al., 1998; Queiroz et al., 2000).

Los efectos adversos de la consanguinidad son consecuencia de que nacen individuos que recibirán de sus padres una mayor proporción de genes que provienen de antecesores comunes lo que producirá una mayor homocigosis (Neira, 1985). Lo anterior implica que 
pueden expresarse genes recesivos de carácter negativo, que muchas veces son letales o bien causan que el individuo sea económicamente indeseable. La expresión de estos genes es baja debido a que son recesivos y por lo tanto se esconden en el estado heterocigótico; por medio del cruce endogámico aparecen en estado homocigótico causando potenciales efectos nocivos (Cassell, 1999). El nivel medio de consanguinidad de los animales en predios lecheros no ha sido medido en Chile, razón por la cual el presente estudio desea hacer un aporte a esta temática.

El objetivo general de este estudio fue cuantificar el grado de consanguinidad en un rebaño lechero de la raza Overo Colorado, en el que se utiliza inseminación artificial (IA) y toros criados en el predio producto de padres de IA. Se analizaron como objetivos específicos la depresión que puedan causar los niveles de consanguinidad sobre la producción de leche y algunos caracteres reproductivos como el lapso interparto y edad al primer parto. Se parte de la hipótesis que el grado de consanguinidad de las vacas del predio analizado no influye negativamente sobre sus rendimientos productivos y reproductivos.

\section{MATERIAL Y MÉTODO}

\section{Materiales}

El estudio utilizó registros de pedigrí de un rebaño de Overo Colorado con un promedio de tres lactancias, y cuya edad fluctuaba entre 2,8 y 15 años, con un promedio de 5,4 años. El predio está ubicado en el cruce Villa Alegre, sector Villa Alegre, comuna de Frutillar provincia de Llanquihue. Todo el rebaño era Overo colorado, ganado de doble propósito (leche y carne) y se utilizaba toros de inseminación artificial (procurando tener en el rebaño no más de 30\% Holstein Friesian rojo) provenientes del Centro de Inseminación Artificial de la UACh y semen de toros importados a través de ANACOC (Asociación de Criadores de Overo Colorado) y de Cooprinsem. Los criterios de selección eran: precio, color (50\% tapado como mínimo), producción de leche, facilidad de parto y lazada. El predio es de aproximadamente 230 has de pradera, 156 vacas en ordeña, edad al primer parto de 30 a 32 meses y distribución de partos 40/60 otoño primavera, respectivamente.

Para el cálculo del coeficiente de endogamia se utilizó el Programa Computacional Pedigree Viewer, desarrollado por Brian Kinghorn del Centro de Evaluación Genética del Ganado de Estados Unidos. Este programa se encuentra disponible de forma gratuita desde la página personal del Dr. Kinghorn (University of New England, 2009).

El programa, lee archivos que tengan extensión ped y extiende toda la estructura de pedigrí en la pantalla. Cada animal está representado por su identidad, o su nombre, o su valor para cualquiera de los caracteres en el archivo de datos. Es posible desplegar, al mismo tiempo, un amplio pedigrí de miles de animales en la pantalla. Los datos se sobreponen en la pantalla, en caso de estos amplios pedigrís, pero en una de las modalidades, la información de un individuo es destacada cuando el cursor del ratón (mouse) pasa sobre él. Es posible poder encontrar uno o más animales por su identidad, por mérito o rango de mérito para un determinado carácter. Se puede calcular coeficientes de consanguinidad y estimaciones BLUP de valores de cría y agregar a la lista de datos que pueden ser desplegados. Sin embargo, existen otras formas de estimar la consanguinidad de ganado lechero como la de Young y Seykora (1996).

\section{Metodología}

Medición de la consanguinidad. De los registros se obtuvieron los datos de pedigrí de las vacas que se encontraban en el rebaño el año 2001 y de los toros que fueron criados en el predio. La información del pedigrí de los toros de inseminación artificial se obtuvo de las empresas que comercializan el semen. En el pedigrí de cada animal, se consideraron cuatro generaciones, es decir, hasta bisabuelos, ya que no existía información que permitiese hacer un análisis de más antecesores. En la Figura 1 se muestra un ejemplo de pedigrí.

Con los datos del pedigrí se elaboró un archivo de 3 columnas en el programa Bloc de Notas, para cada vaca en el rebaño. En la primera se identificó el individuo, en la segunda se identificó el padre y en la tercera la madre (Cuadro 1, con datos utilizados en la Figura 1).

Una vez ordenado el pedigrí de cada vaca como se muestra en el Cuadro 1, se creó el archivo con la extensión *.ped, necesario para correr el programa Pedigree Viewer. Posteriormente, se calcularon individualmente los coeficientes de consanguinidad.

El método que utiliza este programa para calcular el coeficiente de endogamia, es el desarrollado por Wright (1922) citado por Lush (1969), siendo la fórmula:

$$
F_{x}=\sum 0,5^{n_{1}+n_{2}+1}\left(1+F_{A}\right)
$$

Donde,

$F_{x}=$ coeficiente de endogamia del animal $\mathrm{X}$.

$n_{1}=$ número de generaciones desde un padre al antecesor común.

$n_{2}=$ número de generaciones desde el otro padre al antecesor común.

$F_{A}=$ coeficiente de endogamia del antecesor $\mathrm{A}$.

Luego de ingresar los datos el programa construye un diagrama de flechas del pedigrí, identificando cada individuo y diferenciando las líneas paternas y maternas en el color de éstas. 


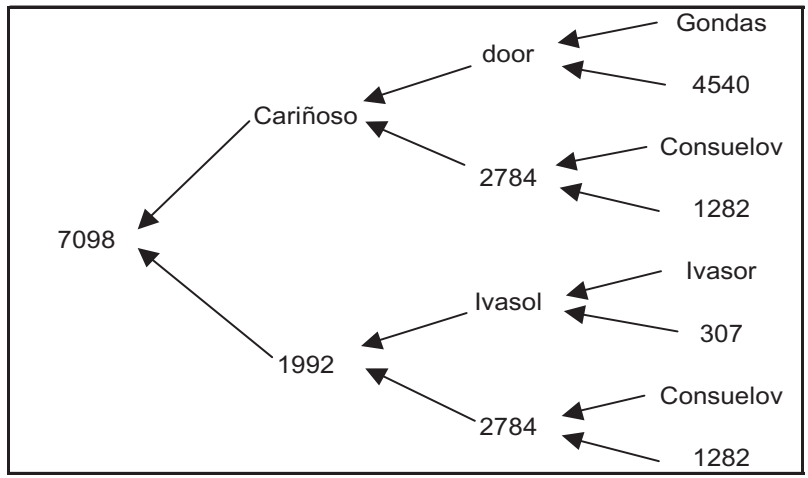

Figura 1. Pedigrí de la vaca 7098.

Figure 1.Pedigree of cow 7098.

Cuadro 1. Ordenamiento de los datos del pedigrí de la vaca 7098, para ingresarlo al Bloc de Notas.

Table 1. Sequence of pedigree data of cow 7098 to be added to the notebook.

\begin{tabular}{ccc}
\hline Identidad & Padre & Madre \\
\hline 7098 & Cariñoso & 1992 \\
Cariñoso & Door & 2784 \\
1992 & Ivasol & 2784 \\
Door & Gondas & 4540 \\
2784 & Consuelov & 1282 \\
Ivasol & Ivasor & 307 \\
2784 & Consuelov & 1282 \\
\hline
\end{tabular}

Lapso interparto. Para evaluar el posible efecto de la consanguinidad sobre esta característica se consideraron 98 vacas, entre los partos de la temporada 2000 y 2001. Se utilizó un Análisis de Varianza con un diseño completamente al azar con distinto número de repeticiones. Para ello, el nivel de consanguinidad correspondió al factor fijo (influyente), considerando como grupo control a las vacas sin endogamia; y las repeticiones, las vacas analizadas.

Edad al primer parto. Para analizar el efecto de la endogamia se utilizó también un ANOVA, con un diseño en bloques completos al azar. Se consideraron bloques a las épocas de nacimiento de las vacas, como una manera de evaluar la existencia de diferencias en su crecimiento según época de nacimiento. Los tratamientos fueron nuevamente los niveles de consanguinidad. Para evaluar esta característica reproductiva se utilizaron 157 vacas.
Producción de leche. Para evaluar el efecto de la consanguinidad en esta característica se corrigieron las lactancias a 305 días, en base a las características de la curva de producción de cada vaca, obteniendo la ecuación de ésta, lo que se realizó en una planilla Excel, para posteriormente obtener la Integral de esta ecuación a 305 días. Las lactancias corregidas a 305 días, se modificaron según la edad de la vaca en base a los antecedentes de Lasley (1970). En las producciones de las vacas corregidas por edad y lactancia de 305 días se consideró el efecto de los toros (padres), debido al hecho que los mejores toros se usan con mayor intensidad, obteniendo por lo tanto mayor descendencia, presentando ésta, en consecuencia, mayor consanguinidad que la de otros toros de menores méritos y mucho menor uso.

Con las lactancias corregidas se procedió a analizar la existencia de diferencias en las producciones según el nivel de consanguinidad que presentaron las vacas, considerando, como control, al grupo de vacas sin endogamia. Para su análisis, se realizó un análisis de varianza con un diseño en bloques completos al azar generalizado; considerando bloques a los grupos de edad y como tratamientos los niveles de consanguinidad. Para este análisis se consideraron 142 vacas. En todos los casos el nivel de significancia fue del $5 \%$.

\section{RESULTADOS Y DISCUSIÓN}

\section{Medición de la consanguinidad}

El programa Pedigree Viewer entrega un diagrama de flechas, indicando el nivel de consanguinidad de cada animal en el pedigrí. En la figura 2 se muestra un ejemplo. En ella se aprecia el pedigrí de la vaca 7098, la cual presentó un $12,5 \%$ de consanguinidad, considerando las cuatro generaciones evaluadas, es decir, hasta bisabuelos.

Por otra parte, el análisis global indicó que de las 157 vacas con antecedentes de pedigrí registrados, sólo un 15,3\% (24 casos) presentó algún nivel de consanguinidad (Cuadro 2).

Además, en el Cuadro 2 se observa que el nivel de consanguinidad varía entre 3,13 y hasta un $25 \%$, con un promedio de $6,12 \%$. Esto corresponde a un coeficiente de endogamia del rebaño completo de $0,94 \%$. Esta cifra es similar a la citada por Queiroz et al. (2000), en Brasil, aunque hay discrepancia en el rango de los niveles de consanguinidad.

Si los datos del presente estudio se comparan con los obtenidos en otros estudios (Wiggans et al., 1995; Thompson et al., 2000a), se constata que en el presente estudio los niveles de consanguinidad fueron inferiores, lo que se debe probablemente al bajo número de animales y de generaciones consideradas en el análisis.

En el Cuadro 3 se presenta la cantidad y proporción relativa de vacas que presentaron distintos niveles de consanguinidad en el rebaño. 


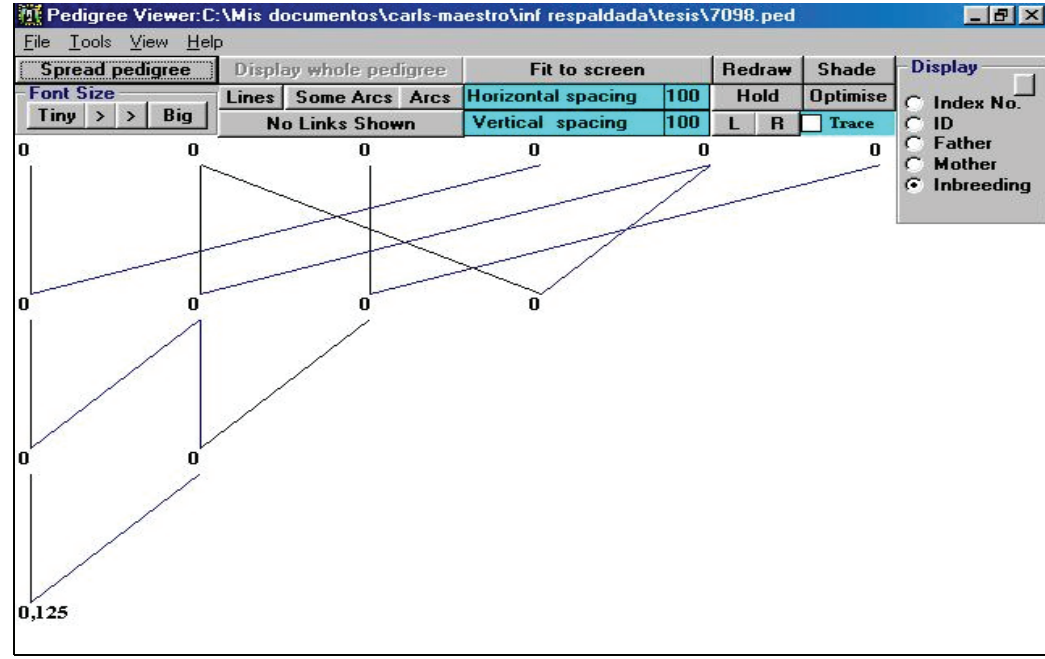

Figura 2. Coeficiente de consanguinidad de la vaca 7098 y de cada animal en el pedigrí.

Figure 2. Inbreeding coefficient of cow 7098 and of each animals in the pedigree.

Cuadro 2. Vacas consanguíneas y sus diferentes niveles de endogamia (F).

Table 2. Inbreeding cows and their different inbreeding level (F).

\begin{tabular}{cccc}
\hline $\boldsymbol{N}^{\boldsymbol{o}}$ vaca & $\boldsymbol{F}(\%)$ & $\boldsymbol{N}^{\boldsymbol{o}}$ vaca & $\boldsymbol{F}(\%)$ \\
\hline 5598 & 3,125 & 4599 & 3,125 \\
5897 & 3,125 & 6797 & 6,25 \\
4494 & 3,125 & 396 & 6,25 \\
3392 & 3,125 & 1099 & 6,25 \\
6597 & 3,125 & 1695 & 6,25 \\
493 & 3,125 & 2099 & 6,25 \\
299 & 3,125 & 395 & 6,25 \\
3999 & 3,125 & 5398 & 9,375 \\
2398 & 3,125 & 896 & 9,375 \\
1498 & 3,125 & 7098 & 12,5 \\
3499 & 3,125 & 6397 & 12,5 \\
4399 & 3,125 & 3295 & 25,0 \\
\hline
\end{tabular}

Cuadro 3. Cantidad y proporción de vacas que presentan los niveles de consanguinidad agrupados en clases.

Table 3. Amount and proportion of cows presenting levels of inbreeding grouped by class.

\begin{tabular}{ccc}
\hline Clases de $\mathbf{F}$ & № vacas & \% vacas \\
\hline $\mathrm{F}=0$ & 133 & 84,7 \\
$0<\mathrm{F}<6,25$ & 13 & 8,28 \\
$6,25 \leq \mathrm{F}<12,5$ & 8 & 5,09 \\
$12,5 \leq \mathrm{F} \leq 25$ & 3 & 1,91 \\
\hline
\end{tabular}

Se observa que la mayoría de las vacas $(84,7 \%)$ no presentó consanguinidad, y que sólo un pequeño porcentaje se encuentra en los niveles más altos ( $\mathrm{F}$ de 12,5 a $25 \%$ ). Esto concuerda con lo señalado por Kuchida et al. (1998) y por Queiroz et al (2000), pero difiere del estudio reportado por Miglior y Burnside (1995), en el cual la mayor proporción de las vacas presentó niveles de consanguinidad del primer rango, es decir, $0<\mathrm{F}<6,25$.

\section{Lapso interparto}

Para cuantificar el posible efecto de la endogamia en éste parámetro reproductivo se consideraron como tratamientos los niveles de consanguinidad, ordenándolos como se indica en el Cuadro 4.

No se detectaron diferencias significativas $(p>0,05)$, indicando que la consanguinidad no afectaría el lapso interparto: Esto discrepa de lo reportado por Cassell (1999), quien obtuvo que por cada $1 \%$ de aumento de la consanguinidad se produce un incremento del lapso interparto de 0,26 días.

Se observa (Anexo 1) que no se detectan diferencias estadísticamente significativas, ya que el $\mathrm{F}$ crítico (Fc) es mayor que el $\mathrm{F}$ calculado $(p<0,05)$, indicando que la consanguinidad no afectaría el lapso interparto: Esto discrepa de Cassell (1999), que obtuvo que por cada $1 \%$ de aumento de la consanguinidad se produce un incremento del lapso interparto de 0,26 días.

\section{Edad al primer parto}

Para evaluar el posible efecto de la endogamia sobre la edad al primer parto los datos se organizaron en bloques según la época de nacimiento de las vacas, considerando tratamientos a los niveles de consanguinidades (Cuadro 5). 
Cuadro 4. Tratamientos según los distintos coeficientes de consanguinidad $(\mathrm{F})$.

Table 4. Treatment depending on different inbreeding coefficients (F).

\begin{tabular}{lc}
\hline Tratamientos & $\begin{array}{c}\text { Consanguinidad } \\
\text { (\%) }\end{array}$ \\
\hline $\begin{array}{l}\text { T1: vacas con coeficiente de consangui- } \\
\text { nidad igual a }\end{array}$ & 3,13 \\
$\begin{array}{l}\text { T2: vacas con coeficiente de consangui- } \\
\text { nidad igual a }\end{array}$ & 6,25 \\
$\begin{array}{l}\text { T3: vacas con coeficiente de consangui- } \\
\text { nidad entre }\end{array}$ & 9,38 y 12,50 \\
T4: testigo & 0,0 \\
\hline
\end{tabular}

Cuadro 5. Tratamientos y bloques en base a la consanguinidad y época de nacimiento de las vacas, respectivamente.

Table 5. Treatment and blocks based on the inbreeding a date of birth, respectively.

\begin{tabular}{cccc}
\hline Bloques & $\begin{array}{c}\text { Época } \\
\text { nac. }\end{array}$ & $\begin{array}{c}\text { Trata- } \\
\text { mientos }\end{array}$ & $\begin{array}{c}\text { Consanguini- } \\
\text { dad (\%) }\end{array}$ \\
\hline B1 & 22 sep - 21 dic & T1 & 3,125 \\
B2 & 22 dic - 21 mar & T2 & $6,25-25$ \\
B3 & 22 mar - 21 jun & T3 & 0,0 \\
B4 & 22 jun - 21 sep & & \\
\hline
\end{tabular}

Los resultados de este análisis (Anexo 2) indica que no se observan diferencias $(p>0,05)$, tanto para bloques como para tratamientos, lo que implicaría que la época de nacimiento y los niveles de consanguinidad no afectan la edad al primer parto.

Este resultado no concuerda con el estudio de Cassell (1999), quien reportó que por cada 1\% de aumento de la consanguinidad, la edad al primer parto se incrementa en 0,36 días. Una posible explicación de esta discrepancia sería el hecho que las vacas consanguíneas son genéticamente superiores a las no consanguíneas del rebaño para esta característica reproductiva. No se observó una interacción entre época de nacimiento y coeficiente de consanguinidad, en relación a la edad al primer parto.

\section{Producción de leche}

Para analizar el efecto de la consanguinidad en esta característica, los datos se agruparon considerando como bloques la edad de las vacas en el rebaño el año 2001 y como tratamientos los niveles de consanguinidad (Cuadro 6).
Cuadro 6. Bloques y tratamientos según edad de las vacas y coeficiente de consanguinidad, respectivamente.

Table 6. Blocks and treatments according to age of the cow and inbreeding coefficient, respectively.

\begin{tabular}{cccc}
\hline Bloques & $\begin{array}{c}\text { Edad } \\
\text { (años) }\end{array}$ & $\begin{array}{c}\text { Trata- } \\
\text { mientos }\end{array}$ & $\begin{array}{c}\text { Consanguini- } \\
\text { dad (\%) }\end{array}$ \\
\hline B1 & $2,00-4,00$ & T1 & 3,125 \\
B2 & $4,01-5,50$ & T2 & $6,25-25$ \\
B3 & $5,51-8,00$ & T3 & 0,0 \\
B4 & 8,01 y más & & \\
\hline
\end{tabular}

Se indica que no existen diferencias $(p>0,05)$ tanto para tratamientos como para bloques (Anexo 3), es decir, la consanguinidad no afectó la producción de leche. No hubo interacción entre edad de las vacas y coeficientes de consanguinidad, en relación a la producción de leche. Estos resultados se diferencian de los presentados por Thompson et al. (2000a y 2000b), que determinaron que la consanguinidad afecta la producción de leche, como también tiene una mayor influencia a edades más jóvenes de las vacas. La diferencia entre el presente estudio y el de Thompson et al. (2000a y $2000 \mathrm{~b}$ ) puede ser atribuida a la superioridad genética de las vacas consanguíneas, lo que está de acuerdo con Cassell (1999), que señala que el cruce consanguíneo no debe evitarse a cualquier costo ya que si la superioridad genética de los animales consanguíneos es mayor que el efecto de la depresión endogámica, se produciría una mejora productiva.

\section{CONCLUSIONES}

Los resultados obtenidos indican que en este rebaño ha habido una selección para mejorar la producción de leche, el lapso interparto y la edad al primer parto; esto habría producido un cierto nivel de consanguinidad en un grupo de vacas, ya que siempre se usaron las mismas líneas paternas. En esta selección se habría logrado contrarrestar el efecto negativo de la consanguinidad por medio del potencial genético de los padres. En estudios a futuro en los cuales se desee medir los niveles de consanguinidad, sería adecuado contar con pedigrís más completos que incluyan más generaciones que las analizadas en este estudio y, por consiguiente, un mayor número de animales. También es importante disponer de información de más características productivas y reproductivas que sean afectadas por la consanguinidad que las analizadas en el presente trabajo, para así tener una mejor apreciación de los distintos efectos que pueda tener la consanguinidad. Indudablemente, ideal sería complementar estos estudios con análisis moleculares. 


\section{REFERENCIAS}

CASSELL, B. 1999. Inbreeding. Publicación № 404-080. http://www.ext.vt.edu/pubs/dairy/404-080/404-080. html \#pdc (acceso, 05.03.2002).

KUCHIDA, K., KAWAHARA, T., SUZUKI, M. MIYOSHI, S. 1998. Relationships between linear type traits and inbreeding coefficients of cow, sire and dam in Japanese Holstein. $6^{\text {th }}$ World Congress on Genetics Applied to Livestock Production, pp. 423-426.

LUSH, J. 1969. Bases para la selección animal. Ediciones Agropecuarias Peri. Buenos Aires.

LASLEY, J. 1970. Genética del mejoramiento del ganado. Editorial Hispano-americana. México.

MACNEIL, M., URICK, J., NEWMAN, S., KNAPP, B. 1992. Selection for postweaning growth in inbred Hereford cattle: The fort Keogh, Montana line 1 example. Journal of Animal Science 70: 723-733.

MIGLIOR, F., BURSIDE, E. 1995. Inbreeding of Canadian Holstein cattle. Journal of Dairy Science 78: 1163-1167.

MUJICA, F. 1992. Hibridismo en producción animal. In: Latrille, L. (Ed.), Producción animal. Universidad Austral de Chile. Facultad de Ciencias Agrarias, Instituto Producción Animal; Serie B - 16. Valdivia, Chile, pp. 179-199.

NEIRA, R. 1985. Introducción al estudio de la consanguinidad en animales. Serie publicación docente № 11. Santiago.
NORTHCUTT, L., BUCHANAN, D., CLUTTER, A. 2001. Inbreeding in cattle. Oklahoma State University. http://www. skally.net/alot/genetic.html (acceso, 12.04.2002).

QUEIROZ, S., ALBUQUERQUE, L., LANZÓN, N. 2000. Efeito da endogamia sobre características de crescimento de bovinos da raca Gir no Brasil. Revista Brasileira de Zootecnia 29(4): 1014-1019.

SMITH, L., CASSELL, B., PEARSON, R. 1998. The effects of inbreeding on the lifetime performance of dairy cattle. Journal of Dairy Science 81: 2729-2737.

THOMPSON, J., EVERETT, R., HAMMERSCHMIDTT, N. 2000a. Effects of inbreeding on production and survival in Holsteins. Journal of Dairy Science 83: 1856-1863.

THOMPSON, J., EVERETT, R., WOLFET, C. 2000b. Effects of inbreeding on production and survival in Jersey. Journal of Dairy Science 83: 2131-2138.

UNIVERSITY OF NEW ENGLAND. 2009. Pedigree Viewer. 6p. http://www-personal.une.edu.au/ bkinghor/pedigree.htm

YOUNG, C., SEYKORA, A. 1996. Estimates of Inbreeding and relationship among registered Holstein females in the United States. Journal of Dairy Science 79: 502-505.

WIGGANS, G., VANRADEN, P., ZUURBIER, J. 1995. Calculation and use of inbreeding coefficients for genetic evaluation of United States dairy cattle. Journal Dairy Science 78: 1584-1590. 


\section{ANEXOS}

Anexo I. Análisis de varianza para evaluar el efecto de la consanguinidad en el lapso interparto.

Attached I. Analysis of variance to evaluate the inbreeding effect during inter-calving period.

\begin{tabular}{cccccc}
\hline FUENTE & GL & SC & CM & F & FC \\
\hline Trat & 3 & $4.099,507$ & $1.366,5026$ & 0,474837 & 2,714 \\
Error & 94 & $270.516,492$ & $2.877,835$ & & \\
\hline Total & 97 & 274.616 & & & \\
\hline
\end{tabular}

Anexo II. Análisis de varianza para evaluar el efecto de la consanguinidad en la edad al primer parto.

Attached II. Analysis of variance to evaluate the inbreeding effect at age of first calving.

\begin{tabular}{cccccc}
\hline FUENTE & GL & SC & CM & F & FC \\
\hline Bloq & 3 & 0,30622 & 0,10207 & 1,94992 & 2,68 \\
Trat & 2 & 0,12035 & 0,06017 & 1,14959 & 3,07 \\
Error exp & 6 & 0,112 & 0,01864 & 0,35614 & 2,17 \\
Error muest & 145 & 7,590 & 0,05234 & & \\
\hline Total & 156 & 8,129 & & & \\
\hline
\end{tabular}

Anexo III. Análisis de varianza para evaluar el efecto de la consanguinidad en la producción de leche.

Attached III. Analysis of variance to evaluate the inbreeding effect on milk production.

\begin{tabular}{cccccc}
\hline FUENTE & GL & SC & CM & F & FC \\
\hline Bloq & 3 & 3094550,944 & 1031516,98 & 1,78765 & 2,68 \\
Trat & 2 & 250065,1975 & 125032,598 & 0,21668 & 3,07 \\
Error exp & 6 & 2083534,748 & 347255,791 & 0,6018 & 2,17 \\
Error muest & 131 & 75589944,41 & 577022,476 & & \\
\hline Total & 142 & 81018095,3 & & & \\
\hline
\end{tabular}


